\title{
COLOMBIA Y ASIA-PACÍFICO: UN EXAMEN EN LA EVOLUCIÓN DE LAS RELACIONES CON ESTA IMPORTANTE ZONA
}

Gloria Milena Torres Rojas ${ }^{1}$

COLOMBIA AND ASIA-PACIFIC: AN ANALYSIS OF THE

EVOLUTION OF RELATIONS WITH THIS IMPORTANT ZONE

COLÔMBIA E ÁSIA-PACÍFICO: ANÁLISE DA EVOLUÇÃO

DAS RELAÇÕES COM ESSA IMPORTANTE REGIÃO

Fecha de recepción: 30 de noviembre del 2020

Fecha de aceptación: 30 de abril del 2021

Disponible en línea: 4 de junio del 2021

Sugerencia de citación. Torres Rojas, G. M. (2021). Colombia y Asia-Pacífico: un examen en la evolución de las relaciones con esta importante zona. Razón Crítica, (11), 183-202. https://doi. org/10.21789/25007807.1697

(1) Docente e investigadora líder del proyecto 2020_029, Sistematización de Experiencias de Rediseño Institucional en Colombia. Escuela Superior de Administración Pública ESAP. Doctora en Derecho Internacional de la Xiamen University, magíster en Análisis de Problemas Políticos e Internacionales de la Universidad Externado de Colombia en convenio con Iheal Paris Docente e investigadora de la Escuela Superior de Administración Pública, Colombia pomarito1981@gmail.com | https://orcid.org/0000-0002-9679-3302 


\section{R E S U M E N}

En este artículo se demuestra que Colombia, a pesar de tener una posición geográfica enclavada en el océano Pacífico, no ha privilegiado una política de alineamiento con Estados Unidos, descuidando así su vínculo con Asia-Pacífico, en comparación con otros países de América Latina que ostentan mayor contacto comercial y político con esa región. Para ello, se recurrió a una metodología cualitativa, desplegada en cuatro partes: en la primera se señalan los antecedentes históricos de las relaciones entre Colombia Asia-Pacífico; en la segunda se describen sus características y problemas subyacentes; en la tercera se indican las ventajas y desafíos, así como las estrategias de Colombia para ingresar en el APEC, y en la cuarta se expondrán las conclusiones del estudio. Estas advierten la precariedad y el rezago de los vínculos entre las regiones, ante lo cual es necesario adoptar estrategias que permitan diversificar los posibles encuentros, no solo en el ámbito comercial, sino también en el cultural y político, de modo que Colombia pueda obtener grandes beneficios.

PALA BRAS CLAVE: Colombia; Asia-Pacífico; evolución; relaciones; balance. 


\section{A B S T R A C T}

This article intends to prove that Colombia, despite its geographic position in the Pacific Ocean, has not favored a policy of alignment with the United States, thus neglecting its relations with the Asia-Pacific region when compared to other Latin American countries with greater commercial and political bonds with that part of the world. For that purpose, a four-phase qualitative methodology was developed. The first phase addresses the historical background of the relations between Colombia and Asia-Pacific; the second describes the characteristics and underlying problems of such relations; the third specifies the advantages and challenges, as well as the strategies designed by Colombia to enter the APEC; the fourth presents the conclusions of this study. Findings warn about the precariousness and lag of the links between both regions, emphasizing the need to adopt strategies that allow diversifying the possible encounters, not only in the commercial sphere but also in the cultural and political dimension, so that Colombia could gain substantial benefits.

\section{Keywords: Colombia, Asia-Pacific, evolution, relations, balance.}

\section{R E S U M O}

Neste artigo, é demonstrado que a Colômbia, apesar de ter uma posição geográfica no oceano Pacífico, não privilegiou uma política de alinhamento com os Estados Unidos, descuidando assim seu vínculo com Ásia-Pacífico, em comparação com outros países da América Latina, que apresentam mais contato comercial e político com essa região. Para isso, recorreu-se a uma metodologia qualitativa, desenvolvida em quatro partes: na primeira, são indicados os antecedentes históricos das relações entre Colômbia e Ásia-Pacífico; na segunda, são descritos características e problemas subjacentes; na terceira, são indicados vantagens e desafios, bem como estratégias da Colômbia para ingressar na Cooperação Econômica Ásia-Pacífico (Apec, em inglês); na quarta, são expostas as conclusões do estudo. Estas advertem a precariedade e a recusa dos vínculos entre as regiões, ante o que é necessário adotar estratégias que permitam diversificar os possíveis encontros, não somente no âmbito comercial, mas também no cultural e no político, a fim de que a Colômbia obtenha grandes benefícios.

PALAVRAS-CHAVE: Colômbia, Ásia-Pacífico, evolução, relações, balanço. 
La política exterior colombiana se ha caracterizado por ser sui generis con respecto a los demás países de América Latina, no solo por la alianza incondicional con Washington, sino también por haber privilegiado la fuerza militar y la seguridad en las relaciones internacionales.

Colombia representa un caso particular, ya que el desarrollo de la capacidad de autonomía no ha sido una prioridad en la política exterior colombiana. La incapacidad del Estado de hacer uso efectivo de la soberanía interna, la debilidad institucional, el vacío de poder estatal en buena parte del territorio, el condicionamiento por el conflicto armado interno y la existencia de diferentes grupos subversivos, son factores que han limitado la política exterior a las condiciones de la política interior (González et al., 2016, p. 271).

Por lo anterior, Colombia ha descuidado, de cierta forma, su inserción internacional en otras áreas, en especial con el Asia-Pacífico, mientras países como Perú, Chile y Ecuador han estrechado más sus vínculos con esta región. Comparado con otros países de América Latina, como Chile, México y Perú, el reconocimiento colombiano de Asia fue tardío porque nuestros referentes históricos son marcadamente occidentales, y por motivos estructurales como el conflicto armado interno.

En este sentido, se demostrará que la inserción de Colombia en el Asia-Pacífico es exigua, por lo menos durante las administraciones que precedieron el gobierno del expresidente Juan Manuel Santos, en el cual sí se percibió el esfuerzo por lograr la diversificación en la política exterior colombiana y mayor cooperación Sur-Sur. No obstante, el progreso reciente de Colombia resulta ínfimo si se compara con el de países como Chile, Argentina, Perú y Brasil, los cuales han logrado una inserción más profunda en Asia, por lo que es necesario que se adopten medidas, no solo 
comerciales, sino también culturales y políticas, para tener mayor injerencia en esta zona, que abriga una de las economías más dinámicas del mundo.

\section{Antecedentes históricos de la relación entre Colombia y Asia-Pacífico}

Pese a que Colombia ha construido una política exterior principalmente alineada a Estados Unidos, no hay que soslayar el que ha interactuado con Asia a través del comercio y las relaciones diplomáticas a partir del gobierno de López Michelsen, cuando se habló de convertir a Colombia en el Japón de Suramérica (Velosa, 2007). Se debe considerar que la relación de Colombia con Asia del este tiene más de un siglo, y comenzó con el Tratado de Amistad, Comercio y Navegación de 1908 con Japón (García, 2011). En este orden de ideas, si bien existe un interés de Colombia por Asia, Colombia ha privilegiado la relación con Japón y, solo recientemente, con el gigante asiático, y ha dejado de lado su relación e interacción con los países miembros de la asean, pese a su posición geográfica privilegiada, por tener límites con el océano Pacífico.

Teniendo presente los antecedentes de la relación entre Colombia y Asia-Pacífico, es menester abordar ahora las características de este nexo.

\section{Características de la relación de Colombia y Asia-Pacífico}

La inserción en la economía del este asiático se ha mostrado insuficiente porque, por un lado, Colombia ha cometido el error de no producir estructuras organizacionales acordes con la importancia de Asia, en especial en el periodo 2002-2010. Por ejemplo, un error notable fue el hecho de haber cerrado las embajadas de Australia, Indonesia y Tailandia a comienzos de la administración de Álvaro Uribe Vélez, para ahorrar costos, según datos de la Cancillería: "se ordenó la reducción de su planta externa para ahorrar alrededor de 20.000 millones de pesos al año" (El Tiempo, 2009), sin calcular los perjuicios, no solo de índole económica, sino también de orden político. Mientras países como Perú tienen embajadas en, India, Indonesia, Japón, Malasia, Nueva Zelanda, Corea del Sur, China, Singapur y Tailandia, y oficinas comerciales en Beijing, Shanghai y Taipéi dentro de la región Asia-Pacífico, Colombia solo cuenta con embajadas en China, Corea, India, Indonesia, Japón, Malasia, Vietnam y Tailandia, lo que ofrece un panorama pobre en las relaciones con los países de la región. 
Asimismo, tal como lo plantea el experto en Asia, Pío García (2011),

existen ciertas trabas que le impiden a Colombia una proyección hacia el Pacífico, porque, en primer lugar, el aparato administrativo, las entidades de planeación y los organismos de control están coordinados alrededor de la política unidimensional de las dos últimas décadas, durante las que ha prevalecido el énfasis en la alianza con Estados Unidos, y en segundo lugar, y es lo fundamental, hay una ausencia de Asia en las prioridades externas del gobierno colombiano (p. 188).

Es de destacar que la Dirección de Asia, África y Oceanía del Ministerio de Relaciones Exteriores de Colombia tiene problemas enormes si se considera que en una misma dirección, estén ubicados dos de los continentes más grandes del mundo, en términos del número de Estados, lo que limita la capacidad de inserción en tal área.

Por otra parte, la tradición diplomática nacional ha hecho que los contactos con Asia sean esporádicos y que no estén articulados en un trabajo conjunto con el sector privado y la academia, como actores fundamentales - en la academia, apenas hasta ahora está surgiendo una comunidad que estudia el Asia-Pacífico-.

Resulta evidente que, antes del actual gobierno del presidente Iván Duque, la sociedad colombiana no había sido consciente de la evolución e importancia de Asia y la inclusión del país en relaciones comerciales, culturales, científicas, técnicas y políticas con ese continente, lo que ha llevado a la

ausencia de contactos de los primeros mandatarios, a la baja participación en la dinámica de cooperación regional, en la cual la participación colombiana es ínfima, a la pérdida de peso institucional del Ministerio de Relaciones Exteriores en los asuntos transpacíficos por su debilidad presupuestal y de representatividad política, y al debilitamiento de los mecanismos de concertación y coordinación de la política transpacífica, este último punto debido a que mecanismos como el colpeEc han venido reduciendo su papel como propulsores de vinculación con Asia-Pacífico (García, 2011, p. 190.)

La anterior afirmación se respalda con el hecho de que Colombia ha sido pasivo históricamente en sus relaciones con Asia y no ha expresado una motivación firme de consolidar lazos y relaciones con aquellos países 
más importantes de la región, como China. De hecho, la embajada de Beijing no tiene para Colombia la misma relevancia que la de Washington, según palabras del exembajador Guillermo Vélez; y, si bien Colombia tiene relaciones diplomáticas con otros países de la región Asia-Pacífico, estos no son prioridad en la política exterior colombiana.

De la misma forma, Colombia no ha sabido aprovechar las relaciones con países del sudeste asiático, pues estableció vínculos tardíos con la Asociación de Naciones del Sudeste Asiático (ASEAn por sus siglas en inglés): solo hasta 1979 se entablaron relaciones diplomáticas con Vietnam y Tailandia; un año después con Indonesia, y luego con Filipinas. Con Singapur se formalizaron vínculos en 1982 y con Malasia, en 1987 (Arcila López de Mesa, 2017). No se puede eludir la importancia que China, Japón y Corea tienen en el escenario mundial, pero tampoco hay que dejar de lado que, para que Colombia tenga una inserción efectiva en la cuenca del Pacífico, debe tener presente que los países de menor desarrollo son también importantes para cumplir con el objetivo de posicionarse en el mundo actual (Velosa, 2007). Lo anterior, porque Colombia no ha tenido un acercamiento significativo en materia comercial con los países del Sudeste Asiático: aunque las exportaciones han crecido —en 2018, se calcularon en 838.200.000 uSD FOB (Legiscomex, 2019)—, las cifras son insignificantes si se comparan con las exportaciones hacia China, las cuales superaron los 4.000.000.000 usd (PROcolombia, 2019).

Según un estudio realizado por Ricardo Coutin (2014), pese a tener un acercamiento con el Sudeste Asiático, Colombia continúa comerciando principalmente con sus socios tradicionales, como Estados Unidos y los países vecinos, con lo cual se posiciona como la economía que menos comercia con Asia. En términos absolutos:

es el país de la Alianza del Pacífico que menos exporta al grupo de países ASEAN+5, y es el penúltimo exportador a China, Japón y Corea del Sur; en términos porcentuales, su desempeño es similar. En teoría las economías son complementarias; en la práctica existen unas relaciones comerciales incipientes (Coutin, 2014, pp. 122-123).

Esto muestra la escasa importancia que se le ha dado, por parte de los gobiernos, al Sudeste Asiático, por lo que

el intercambio comercial de Colombia con el Sudeste Asiático es insignificante tanto en el comercio global de Colombia como en el de la 
región, por lo que se hace necesario una mayor presencia, y sobre todo un mayor trabajo mancomunado, entre el gobierno nacional, el servicio exterior, los empresarios y los grupos de interés para poder cambiar las cifras mencionadas antes. Pero para poder hacerlo de una manera efectiva y eficiente, es necesario reforzar la estructura organizacional presente en el sudeste (Velosa, 2007, p. XX).

Lo anterior refleja que la interacción de Colombia con países del Sudeste Asiático es precaria. En concreto, solo se ha aproximado con países de la ASEAN en temas económicos que se restringen a memorandos de entendimiento aún muy incipientes, como el que actualmente tiene con Malasia en programas de control de contenedores, compra de semillas y transferencia de tecnología (Cancillería de Colombia, 2019). Se han obviado temas políticos que también son pertinentes, máxime cuando Colombia tiene como prioridad ingresar al Foro de Cooperación Económica del Asia-Pacífico (APEC, por sus siglas en inglés) desde mayo de 1995, lo cual llevó a que Colombia fuera invitado a los grupos de trabajo de este Foro; empero, Colombia privilegió su internacionalización por medio de acuerdos como la Comunidad Andina (CAN), el Mercado Común del Sur (Mercosur) y las negociaciones de la Cumbre de Doha en las negociaciones del TLC con Washington, lo que, a la postre, implicó que su relación con Asia-Pacífico perdiera relevancia.

En relación con el dragón asiático, Ladino y Prieto (2011) plantean que

Los acercamientos que se han producido recientemente con China son importantes y Colombia debe intensificar esfuerzos en este sentido. China promete ser la mayor potencia económica mundial en el plazo de unos 20 o 30 años, pero Colombia ha llegado tarde al establecimiento de acuerdos profundos de cooperación económica con este gigante asiático, en comparación con vecinos de nuestra región como Brasil, Perú y Chile.

Lo anterior se evidencia, por ejemplo, en que China y el Gigante Suramericano establecieron el Plan de Acción Conjunta 2015-2021, el Plan de Cooperación por Diez Años (2012-2020), así como el establecimiento del Comité de Coordinación y Cooperación de Alto Nivel China-Brasil (Cosban) (Busilli, 2017); y Perú no solo tiene un TlC con el dragón asiático, sino que también participa en la iniciativa Una Franja, una Ruta (One Belt 
One Road) y tiene un comercio bilateral con Beijing, que asciende a los 20.000.000 uSD (Agencia EFE, 2019).

Es de observar que, si bien los empresarios nacionales le dan trascendencia a China, como se demuestra en el hecho de que empresas colombianas - como Linen Traders (bebidas y café), Grupo TAP (café y cacao), The Colombian Factory (café), Porkcolombia y cı Porkco (carne de cerdo), Buencafé (café liofilizado), Agrofrut (pulpas de fruta) y Basal Trading - han gestionado negocios con este, solo enfocan sus relaciones desde el punto de vista de las exportaciones de bienes tradicionales, o commodities, mas no sobre productos de mayor valor agregado, es decir, sin pensar en posicionarse en el mercado chino con productos que involucren mayor innovación y niveles de conocimiento, de ahí que la balanza nacional de negociaciones con ese país sea deficitaria.

En consecuencia, pese a que Colombia se ha beneficiado por sus exportaciones de materias primas a China - en 2018, el total de las exportaciones a China fue de 4.172.700.000 UsD (El Tiempo, 2019)—, es necesario que se diversifique e incursione en esferas del sector terciario y en bienes de capital, que son los que aportan mayores ganancias en el comercio internacional, pues su dependencia de las materias primas y commodities sería contraproducente a largo plazo, toda vez que podría darse la denominada enfermedad holandesa, en la que entran divisas, se valoriza la moneda nacional, pero se produce una pérdida de competitividad de la economía y los otros rubros de exportaciones caen. Si Colombia incrementa su dependencia en el mercado de commodities y en los precios de materias primas, sería nocivo, ya que en el evento en que el precio de las materias primas caiga, la economía colombiana se derrumbaría.

El problema que ha tenido Colombia en su inserción económica mundial, en especial con Asia-Pacífico, es que ha sido incipiente, si se advierte cómo sus exportaciones se han sustentado en productos básicos como commodities, en productos energéticos y de baja tecnología, sin mayor valor agregado. De esta manera, como lo afirma Diego Cardona (2011):

lo peor que puede sucederles a los países latinoamericanos es que los precios de las materias primas continúen siendo altos; ello porque la ganancia fácil a corto plazo haría olvidar las necesidades de enfatizar en las industrias de transformación que requieren mayor valor agregado y competitividad. 


\section{Ventajas y desafíos de la relación entre Colombia y Asia-Pacífico}

Según la Cepal (2010), la relación comercial entre América Latina y Asia ofrece oportunidades y desafíos, por lo que es urgente evitar que se dé un comercio de tipo "centro-periferia”, en el que Asia, especialmente China, sería el centro y los países de la región, la nueva periferia. Este panorama desalentador podría superarse solo si los países de la región, entre ellos Colombia, ejecuten estrategias basadas, no solo en ventajas comparativas, sino también en ventajas competitivas en el comercio internacional: en innovación y valor agregado en los productos, en investigación, educación y desarrollo. Si bien es cierto que la exportación de Colombia a Asia-Pacífico no es muy alta si se compara con la de los demás países de la región, "desde el año 2006 se ha duplicado, pasando de 3.53 a 6.06 millones de dólares en 2012” (Tremolada, 2015, p. 727). Así es como, en 2018, se compararon cuatro países de la Alianza del Pacífico y se concluyó que Colombia posee el nivel más bajo de exportaciones hacia Asia-Pacífico; de hecho, para el 2018, escasamente alcanzaba a exportar 2.000.000.000 uSD, cifra ínfima comparada con países como Chile y Perú (Bula, 2019).

La importancia del aspecto económico es innegable, pero se considera que la inserción en Asia no debe limitarse a este, por lo que se comparte la opinión de Eduardo Velosa (2011) cuando expresa:

Pero, más allá de los negocios, ¿no valdría la pena hablar de otras cosas?; ¿tener objetivos adicionales? Se me ocurre, por ejemplo, que con Japón se podría hablar, de la reforma a Naciones Unidas, de cooperación técnica, de medio ambiente basado en energía limpia, de transferencia de tecnología, de trata de personas... Con Corea, de la política de claridad, de desarrollo colectivo, de alianzas sectoriales, de "marca país", de infraestructura, de experiencias no militares de seguridad, de la posición de Colombia con respecto a Corea del Norte, entre otros.

Lo anterior demuestra que se debe ampliar la perspectiva al establecer relaciones con Asia, pues los objetivos de Colombia no deben ser solo comerciales, porque

Asia es más que economía, si bien son importantes los temas comerciales, hay otros temas y factores, hay temas políticos con los que se puede trabajar, porque hay, por ejemplo, fijación de Colombia por 
ingresar a la APEC y ya se cuenta en parte con el apoyo de Corea, pero Colombia sólo incluyó dos países, ignorando a los países de la ASEAN, países muy importantes en nuestra aspiración de ingresar a la APEC (sic).

Además, el Ministerio de Comercio y el Ministerio de Cultura deben reposicionar a Colombia en la agenda de China, desde las perspectivas latinoamericana y asiática, y este objetivo, desafortunadamente, no se lo ha planteado el actual Gobierno. Esto es de suma importancia para Colombia, no solo en relación con China, sino también con otros países claves, como India, porque "los acuerdos bilaterales de inversión hasta ahora firmados con China e India no tendrán ningún efecto a menos que estén acompañados de una fuerte ofensiva promocional y se basen en ese compromiso político que nos ha faltado" (Uribe, 2010).

Un paso en el reposicionamiento colombiano en la agenda del gigante asiático es, como sugiere Zheng Bingwen (2012), "fortaleciendo los intercambios humanísticos que ayudan a impulsar contactos amistosos y cooperación” (p. 32). En este aspecto, es importante que Colombia promueva su cultura e incremente su poder blando frente al dragón asiático a través de diferentes medios, tales como el aumento de intercambios académicos o el establecimiento de observatorios de investigación que propendan por eliminar dudas respecto a China, como el "nuevo colonialismo chino", la "amenaza china" o "la trampa de la deuda", refiriéndose a la iniciativa de la Franja de la Ruta, que, para algunos, tiene como fin incursionar en la región con el ánimo de luchar por la hegemonía con Washington y para endeudar a los países latinoamericanos. Estas teorías han sido ideadas, en su mayoría, por académicos occidentales y por países como Estados Unidos. Un ejemplo de cómo académicos occidentales se refieren a la presencia de China en Latinoamérica está la afirmación de Cristina de Esperanza (2019), quien aduce que

\footnotetext{
Desde principios de siglo, China ha redoblado su presencia económica y política en América Latina. Ahora que la competición estratégica entre EE. UU. y China se recrudece, esta situación podría acabar convirtiendo Latinoamérica en el nuevo campo de batalla para la lucha hegemónica entre EE. UU. y China.
}

Otro ejemplo de esas críticas temerarias contra China es la afirmación de que "la Nueva Ruta de la Seda no solo representa un audaz 
aumento de la influencia geopolítica y estratégica de China, sino también el endeudamiento de los países receptores" (BBC News, 2019).

Estas afirmaciones desconocen que China no está compitiendo con Estados Unidos en América Latina, sino tan solo invitando a la región a una iniciativa de cooperación, como lo es la Franja de la Ruta de la Seda, la cual implica ganancias para ambas partes y no un endeudamiento de los países receptores, ya que China establecerá mecanismos para eludir eventuales problemas de deuda en los países que hagan parte de este proyecto. Es importante señalar que los cuestionamientos occidentales con respecto a la presencia de China en América Latina no tienen asidero alguno y no han impedido que China incremente sus vínculos con la región y la incluya en la iniciativa de la Nueva Ruta de la Seda como una "extensión natural de su iniciativa".

No obstante los avances de China en la región, no hay que olvidar que el posicionamiento colombiano en la agenda del gigante asiático se reforzaría si se acepta la invitación de Beijing a construir de manera conjunta la Franja de la Ruta, a fin de obtener ventajas recíprocas que interconectarían a ambos países, máxime si se tiene en cuenta la ubicación privilegiada de Colombia en América Latina.

\section{Estrategias para la inserción de Colombia en el APEC}

Es necesario aplicar estrategias de inserción de Colombia no solo en China, sino también en el Sudeste Asiático para lograr su ingreso en el APEC. Es menester, entonces, tener en cuenta que la ASEAN es una organización particular en la cual lo que se denomina ASEAN way impone ciertos retos y estrategias que no pueden ser utilizados en otros escenarios de integración, especialmente porque Colombia no está preparada para hacer frente a problemas como su informalidad, es decir, a la ausencia de instituciones fuertes que regulen las acciones del Estado. Además, el tema del consenso es fundamental, y por eso la interacción con países del Sudeste Asiático se debe pensar en términos de la ubicación estratégica, ya que no es posible tener una embajada en los 11 países, pero sí en los países más representativos de la MP, que son los miembros fundadores, como Indonesia, Malasia, Singapur, Filipinas y Tailandia.

Con Filipinas hay una ventaja, y es el pasado histórico común por el hecho de que es un país hispanodescendiente. A partir de ello, se puede observar cómo se relaciona con los demás países de la región, no obstante estar en medio de culturas budistas o musulmanes; o sea, ver cómo hacen para lidiar con esas circunstancias, sabiendo que Filipinas no 
es homogéneo. Asimismo, el caso de Tailandia es importante estudiarlo en relación con el problema de las drogas, para analizar su experiencia en la erradicación de cultivos ilícitos, pues tenía el problema del opio, pero hoy no se reconoce como productor de heroína, a pesar de que se sigue consumiendo, sin perjuicio del problema de vecindad que tiene frente a Laos, con las implicaciones de todo su sistema político. Esto resulta conveniente para Colombia al proporcionar opciones diferentes a las fumigaciones y aspersiones indiscriminadas, que implican problemas medioambientales y de salud para las poblaciones. En ese orden de ideas, estas lecciones o acercamientos no comerciales son importantes para demostrar un interés real de Colombia en insertarse en aquella región.

Malasia y Singapur son importantes por sus experiencias de crecimiento económico, especialmente en cuanto al tema portuario y de infraestructura, si se tiene en cuenta que Singapur tiene uno de los niveles de vida más altos de la región, lo que lo convirtió en un atractivo para el comercio internacional. Si Colombia quiere convertirse en la bisagra comercial de América Latina, la experiencia de Singapur ilustra cómo volverse un paso obligado y obtener réditos económicos de ello.

La inserción de Colombia en Asia, se reitera, es precaria si se compara con otros países de la región, como Perú, Chile y México, los cuales, con más continuidad y mayor anticipación, establecieron vínculos con el Pacífico. A modo de ejemplo, "las exportaciones peruanas a Japón duplican las de Colombia, mientras que las de México y Chile fueron ocho y diez veces mayores respectivamente" (Lara, 2007, p. 237). Por otro lado, mientras que Colombia no ha logrado ingresar al APEC, Chile ya lo hizo,

lo cual le ha permitido grandes ventajas como permitir a las autoridades de Chile reunirse con sus pares para tratar temas que van más allá de la agenda del foro, esta cercanía le ha proporcionado a Chile, el escenario para iniciar, avanzar y anunciar la suscripción de tratados de libre comercio con muchas economías miembros de APEC, incluyendo, Australia, Canadá, Estados Unidos, Corea, Perú, China, Japón los miembros del denominado acuerdo estratégico de Libre Comercio Transpacífico entre cuatro economías APEC P4. Más aún, Chile está negociando acuerdos de libre comercio con Malasia y Vietnam, y ha realizado estudios de factibilidad con Tailandia, y en ejecución con Hong Kong e Indonesia (Ministerio de Relaciones Exteriores de Chile, 2020). 
Mientras que México ha establecido vínculos diferentes a los comerciales en Asia-Pacífico, Colombia solo se ha limitado a temas comerciales, como la firma del Acuerdo para la Protección Recíproca de Inversiones y el inicio del estudio conjunto del Acuerdo de Asociación Económica con Japón, y la aceleración del TLC y la firma de cinco acuerdos de cooperación económica con Corea (El Heraldo, 2011).

Debido a que la inserción de Colombia en Asia-Pacífico es restringida, es necesario tomar en cuenta algunos de los planteamientos que expone Pío García (2011), en el sentido de
generar capacidad institucional, rediseñando el colpecc, en aras de que este pueda seguir la participación colombiana en instancias como el PECC, el PBEC, la APEC y la FOCALAE, la formación de recursos humanos, con la especialización de profesionales de la carrera diplomática en asuntos asiáticos, la generación de información permanente sobre los asuntos asiáticos y la gestión diplomática de alto nivel, con encuentros semestrales en uno y otro lado del Pacífico (sic).

En suma, a pesar de que la inserción de Colombia en Asia no es un elemento nuevo, sino que ha sido considerado por diversos gobiernos, los esfuerzos emprendidos han sido insuficientes en comparación con otros países de la región. De ahí que se afirme que la inclusión de Colombia en esa importante zona es precaria, porque, no obstante las numerosas iniciativas y despliegues intermitentes,

nuestra política exterior no ha logrado del todo trascender la simple expresión de intenciones de acercamiento a esa rica y prospera región. Aún no se ha podido estructurar una verdadera política estatal o, al menos, una estrategia articulada de mediano o largo plazo desprovista de los vaivenes de los distintos gobiernos (Yumpu, 2010).

\section{CONCLUSIONES}

- Con respecto a la inserción económica de Colombia en Asia, se concluye que, con base en el informe de la Cepal, el desempeño exportador del país no ha sido suficiente para vincularse comercialmente en la economía global, en especial con la región 
Asia-Pacífico. La inclusión de Colombia en Asia-Pacífico es precaria porque el comercio solo representa el $12 \%$ del total de las exportaciones, mientras que, para otros países, como Chile, representa el $49 \%$ (3.333.000.000 usD), y, para el Perú, $28 \%$. En el caso de Ecuador, su comercio con la ASEAN pasó de 97.000.000 USD a 140.000.000 uSD, según datos del Fondo Monetario Internacional.

- Colombia no ha logrado progresar en su introducción comercial mundial, aunque tenga un TLC con Estados Unidos y suscritos 15 acuerdos comerciales (PROCOLOMBIA, 2019). Las ventajas del TLC y los acuerdos no han sido absolutas por las asimetrías existentes en las economías con estos países, máxime cuando Colombia carece de competitividad y un capital humano idóneo que pueda competir con países más desarrollados.

- Si bien algunos países de América del Sur han mostrado un mayor dinamismo exportador, esto está ligado a factores externos como el renovado impulso de la demanda internacional de materias primas y el consiguiente aumento de sus precios. Es por esta razón que, a fin de que Colombia pueda mejorar su inserción internacional, es necesario que haga esfuerzos para obtener una diversificación en sus exportaciones, aumentar su competitividad y fortalecer la cooperación regional en los ámbitos de la infraestructura, la logística y el comercio intrarregional.

- Según Eduardo Veloza, no hay que privilegiar un foro sobre otro en la institucionalidad transpacífica porque, no solo hay que priorizar el aspecto económico, sino también la infraestructura, las telecomunicaciones y el desarrollo de género en los grupos de trabajo en los distintos foros, como el APEc, el Focalae y el P4, en los que Colombia no solo sea receptora, sino también proponente en temas de su interés.

Si bien hay desafíos en la relación de China con algunos países de América Latina, no hay que soslayar que, como lo señalan Jiang Shixue China también ofrece nuevas oportunidades, porque, aunque el comercio entre América Latina y Beijing se centra en productos básicos, el hecho de que China necesite materias primas de América Latina también representa beneficios para la región. 
Se comparte la opinión de autores como Yang Fuchan frente a que el resurgimiento chino es positivo para la comunidad internacional y trae oportunidades, aunque también presenta desafíos. Oportunidades como la futura participación en la iniciativa de la Franja y la Ruta, ya que, aunque la región no tiene una participación formal en la misma, de acuerdo con lo expresado por el ministro de Asuntos Exteriores chino, Wang Yi (2018), "América Latina constituye una extensión natural y un participante indispensable de la iniciativa".

Es indispensable que Colombia tenga en cuenta que, si bien la demanda de materias primas por parte de China e India es algo beneficioso porque contribuye al auge exportador colombiano, este éxito podría anestesiar toda voluntad de diversificación más allá de las materias primas (Santiso, 2006), por lo que Colombia debe imprimirle mayor valor agregado a sus exportaciones, tal como lo hicieron Chile, Brasil y México, y buscar no solo depender de ventajas comparativas, basadas en recursos naturales y energéticos, sino irrumpir en renglones de exportación basados en mayor innovación, tecnología y valor agregado, para no quedar rezagados en la economía mundial.

- $\quad$ Es prioritario que Colombia alcance acuerdos comerciales y un mayor involucramiento político y económico con Asia-Pacífico. Sin embargo, no solo son necesarios vínculos económicos más fuertes, sino también esfuerzos políticos para lograr mayor representación diplomática en los países del Sudeste Asiático, que presentan una fisonomía similar a América Latina: zona rica en recursos naturales y humanos. Además de los vínculos bilaterales, son convenientes más tratos grupales: Vietnam y Tailandia son países con los cuales Colombia debe interactuar, tanto como con Malasia, Singapur e Indonesia.

- $\quad$ Se requiere una política exterior más intensiva y equilibrada en la relación con Asia-Pacífico, a pesar de los esfuerzos del presidente Duque para no dejar perder de nuevo el tren asiático, porque quizás sea la última oportunidad en este empeño.

- Lo más importante que necesita Colombia para involucrarse en Asia es dejar de lado su "parroquialismo" en política exterior y darse cuenta de que el mundo cambió, de que, tal como lo señala el Informe de la Misión de Política Exterior, "estamos presenciando una redistribución del poder de occidente a 
oriente, la erosión de la hegemonía estadounidense, mayor autonomía de los países de la región con respecto a Estados Unidos, así como nuevos liderazgos en América Latina” (Bell et al., 2010, p. 9). Por ello, es imprescindible que la política exterior se adapte a las nuevas realidades y dinámicas del entorno regional e internacional. Un paso correcto en esta dirección sería la incorporación de Colombia en la Iniciativa de la Franja y la Ruta China y el abandono de la política de alineamiento con Washington, ya que ello le proporcionaría beneficios no solo en infraestructura e inversiones, sino también de conectividad con países asiáticos que ya hacen parte de la Iniciativa.

- Colombia debe mirar a la región del Asia-Pacifico, en especial a China, no como un enigma, sino como una oportunidad de diversificar sus relaciones en condiciones de igualdad y no de sometimiento a un hegemon, como tradicionalmente lo ha hecho con su política de respice polum o "mirar hacia el norte". Países de la región, como Perú, Chile y Brasil, tienen nexos más fuertes con el gigante asiático que con Washington, lo que les ha proporcionado grandes beneficios. Un ejemplo de ello es que, según datos del Sunat, entre 2018 y 2020, las exportaciones peruanas con destino a China registraron un crecimiento del $143 \%$ (Confiep, 2019).

- Un paso en este sentido es forjar una política unificada con los países vecinos, en la cual se le dé trascendencia a la diversificación de la oferta exportadora para aumentar la competitividad y la implantación de cadenas de valor.

- $\quad$ Finalmente, surge una pregunta para futuras investigaciones, a saber: ¿cómo lograr que Colombia y los países de América Latina consigan una inserción positiva, duradera e igualitaria con la región del Asia-Pacífico, que implique, a largo plazo, una comunidad de destino compartido y la construcción de un nuevo orden global acorde con las necesidades y realidades del siglo xxi? 


\section{REFERENCIAS}

Agencia Efe. (2019, 24 de enero). Perú y China negociarán a partir de abril mejoras en su acuerdo comercial. Agencia EFE. https://www.efe.com/efe/ america/economia/peru-y-china-negociaran-a-partir-de-abril-mejoras-en-suacuerdo-comercial/20000011-3877459

Arcila López de Mesa, N. (2017). Estrategias de la política exterior colombiana del siglo XXI para la inserción Asia-Pacifico. http://repositorio.esumer.edu.co/bitstream/ esumer/1370/2/Esumer_politica.pdf

ввс. (2019, 26 de abril). Los países de América Latina que forman parte de la Nueva Ruta de la Seda de China. BBC. https://www.bbc.com/mundo/noticiasamerica-latina-48071584

Bell, G., Borda, S., Gómez, H. J., Ramírez, S., Reina, M., Reyes, C, \& Tokatlian, J. G. (2010). Misión de política exterior de Colombia. Ministerio de Relaciones Exteriores, Fedesarrollo, Banco Interamericano de Desarrollo y cAF. https://www.repository.fedesarrollo.org.co/bitstream/handle/11445/228/ Mision\%20de\%20Politica\%20Exterior-\%20Informe\%20final. pdf? sequence $=2 \&$ isAllowed $=\mathrm{y}$

Bingwen, Z. (2012). Sesenta años de relaciones entre China y América Latina. En B. Creutzfeldt (Ed.), China en América Latina (p. 83). Universidad Externado de Colombia.

Bula, J. I. (2019, 15 de julio). De espaldas al Pacífico: las relaciones comerciales entre Colombia y China. UN Periódico Digital. https://unperiodico.unal.edu. co/pages/detail/de-espaldas-al-pacifico-las-relaciones-comerciales-entrecolombia-y-china/

Busilli, V. (2017). La alianza estratégica entre Brasil y China y sus relaciones económicas bilaterales. 9. ${ }^{\circ}$ Congreso Latinoamericano de Ciencia Política. Montevideo, Uruguay. http://www.congresoalacip2017.org/arquivo/downloadpublic2?q=YToyOntzOjY6InBhcmFtcyI7czozNToiYToxOntzOjEwOiJJRF9BUlFVSVZPIjtzOjQ6IjIyNjAiO30iO3M6MToiaCI7czozMjoiYWU0ZjViMDNhYTMxYTExYTM4YTVlMjc0ZGY3MmRiMDQiO30

Cancillería de Colombia. (2019, 8 de julio). Colombia firma memorando de entendimiento con Naciones Unidas para la implementación del programa global de control de contenedores. Cancillería de Colombia. https:// www.cancilleria.gov.co/newsroom/news/colombia-firma-memorandoentendimiento-naciones-unidas-implementacion-programa-global

Caracol Radio. (2019, 24 de julio). China es el futuro para las exportaciones colombianas. Caracol Radio. https://caracol.com.co/radio/2019/07/24/ economia/1563988324_405352.html

Cardona, D. (2011). ¿Puede tener Colombia una estrategia de política exterior? Diego Cardona Editor. 
Cepal. (2010). Panorama de la Inserción Internacional de América Latina y el Caribe 2010-2011: la región en la década de las economias emergentes. Cepal. https:// www.cepal.org/es/publicaciones/1180-panorama-la-insercion-internacionalamerica-latina-caribe-2010-2011-la-region-la

Confiep. (2019, 27 de marzo). TLC con China: Estos son los beneficios tras 9 años de su entrada en vigencia. Confiep. https://www.confiep.org.pe/noticias/economia/ tlc-con-china-estos-son-los-beneficios-tras-9-anos-de-su-entrada-en-vigencia/

Coutin, R. (2014). La inserción al Asia-Pacífico, oportunidades y desafíos para el Valle del Cauca y la región pacífica colombiana. CS, (14), 111-140. https://doi. org/10.18046/recs.i14.1852

El Heraldo. (2011, 16 de septiembre). Presidente Santos regresa con importantes logros de su gira por Japón y Corea. El Heraldo. https://www.elheraldo.co/ nacional/presidente-santos-regresa-con-importantes-logros-de-su-gira-porjap-n-y-corea-37911

El Tiempo. (2019, 1 de octubre). Colombia - China, una relación comercial que continúa en crecimiento. El Tiempo. https://www.eltiempo.com/ mas-contenido/colombia-china-una-relacion-comercial-que-continua-encrecimiento- 416268

Esperanza, C. (2019, 10 de marzo). China en América Latina: ¿el inicio de una nueva Guerra Fría? ЕOM. https://elordenmundial.com/china-en-americalatina-el-inicio-de-una-nueva-guerra-fria/

Fuchang, Y. (2008). Contemporary China. World Affairs press.

García, P. (2011). Colombia transpacífica: opciones de política y liderazgo regional. http://library.fes.de/pdf-files//bueros/kolumbien/08196.pdf

García, P. (coord.) (2005). ¿Es deseable el ingreso de Colombia a APEc? Policy Paper, (17), 1-8. https://library.fes.de/pdf-files/bueros/kolumbien/50476.pdf

González, C., Mesa Bedoya, J., Londoño, G. Política Exterior Colombiana 20102014, ¿Giro a la Autonomía? Revista de Relaciones Internacionales Estrategia y Seguridad, 1(11) 207-291

Hsiang, A. (2010). China Rising in Latin America: More Opportunities than Challenges. Journal of Emerging Knowledge on Emerging Markets, 1(4). https:// doi.org/10.7885/1946-651X.1003

Ladino, N., \& Prieto, G. (2011, 19 de septiembre). La gira de Santos por Asia y el “despertar de un sueño”. Razón Pública. https://razonpublica.com/la-gira-desantos-por-asia-y-el-despertar-de-un-sueno/

Lara, A. (2007). Aproximación práctica a las relaciones con Asia. Fundación ColomboCoreana y Fescol.

Legiscomex. (2019). El mercado colombiano enfoca su mira en el Sudeste Asiático. Legiscomex. https://www.legiscomex.com/Documentos/mercado-colombianomira-sudeste-asiatico-legiscomex

Ministerio de Relaciones Exteriores de Chile. (2020). Embajada de Chile en Colombia. Ministerio de Relaciones Exteriores. https://chile.gob.cl/colombia 
Procolombia. (2019, 10 de junio). Colombia es un mercado clave para hacer negocios con Japón. Procolombia. https://procolombia.co/noticias/colombiaes-un-mercado-clave-para-hacer-negocios-con-japon

Santiso, J. (2006). ¿Realismo mágico? China e India en América Latina y África. Economía Exterior, 2006(38), 59-69. https://www.oecd.org/dev/38447180.pdf

Shixue, J. (2006). Una mirada China a las relaciones con América Latina. https:// nuso.org/articulo/una-mirada-china-a-las-relaciones-con-america-latina/

sice. (2020). Acuerdo Marco para una Asociación Económica más Cercana entre el Gobierno de la República del Perú y el Gobierno del Reino de Tailandia. sice. http://www.sice.oas.org/Trade/PER_THA_FTA/framework_s.asp

Tremolada, E. (2015). ¿La Alianza del Pacífico facilita la inserción de Colombia en la región Asia-Pacífico. Papel Político, 19(2), 721-752. https://doi. org/10.11144/Javeriana.PAPO19-2.apfi

Uribe, J. (2010, 19 de septiembre). La política colombiana hacia el Asia-Pacífico. OVAP. https://www.utadeo.edu.co/es/notas/observatorio-asia-pacifico/142591/ la-politica-colombiana-hacia-el-asia-pacifico

Velosa, E. (2007). Asia Hoy. Fescol.

Velosa, E. (2011). Del Asia viene un Santos cargado de... Semana. https://www. semana.com/opinion/articulo/del-asia-viene-santos-cargado-de/246675-3

Wang, Y. (2018). América Latina, la nueva escala en la Ruta de la Seda del siglo xx. https://www.chinaenamericalatina.info/america-latina-la-nueva-escala-en-laruta-de-la-seda-del-siglo-xxi/

Yumpu. (2010). 2010, un año de intensa actividad de México en Asia-Pacífico. Yumpu. https://www.yumpu.com/es/document/view/19013695/2010-un-anode-intensa-actividad-de-mexico-en-asia-pacifico 